\title{
GESTIÓN Y FINANCIAMIENTO DE LAS INVESTIGACIONES POR EL INSTITUTO NACIONAL DE SALUD, PERÚ 2004-2008
}

\author{
Gladys Garro ${ }^{1, a}$, Henry Mormontoy ${ }^{1, b}$, Martín Yagui ${ }^{1, c}$
}

\begin{abstract}
RESUMEN
Se analizó los resultados de los proyectos de investigación que han sido aprobados y financiados por el Instituto Nacional de Salud durante el periodo 2004-2008. De 182 investigaciones aprobadas y presupuestadas, se ejecutaron 150 (82\%); $86 \%$ (129/150) culminaron en informe final y solo $14 \%$ (18/129) se publicaron en revistas indizadas, el promedio de tiempo de publicación de un artículo fue de 2,7 años. De las investigaciones presentadas, 68 (45\%), fueron a través del fondo concursable, $60(40 \%)$ institucionales, $14(9 \%)$ de direcciones regionales de salud y $8(5 \%)$ colaborativas. El presupuesto ejecutado fue de $\$ 5032906,62$. En promedio, se asignó a cada investigación $\$ 33552,71$ y el costo por cada publicación fue de $\$ 279605,92$; la distribución del presupuesto según objeto o tema de estudio fue $61 \%$ para enfermedades transmisibles, $12 \%$ para no transmisibles y $27 \%$ para desarrollo tecnológico. La promoción, desarrollo y financiamiento de la investigación en el Instituto Nacional de Salud durante este periodo, ha tenido una tendencia descendente, influenciada por la política institucional. Para revertir esta situación no solo a nivel institucional sino nacional, es necesario que el Estado defina su política nacional de investigación, respetando las prioridades nacionales y regionales de investigación en salud.
\end{abstract}

Palabras clave: Fuentes de financiación de investigación; Financiación gubernamental; Investigación biomédica; Gestión de ciencia, tecnología e innovación en salud; Perú (fuente: DeCS BIREME).

\section{MANAGEMENT AND FUNDING OF THE RESEARCH BY THE PERUVIAN NATIONAL INSTITUTE OF HEALTH, 2004-2008}

\begin{abstract}
The results of the research projects that have been approved and funded by the Instituto Nacional de Salud (Peru) during the period 2004-2008 were analyzed. Out of 182 approved and funded research projects, $150(82 \%)$ were actually performed, $86 \%(129 / 150)$ ended in the final report and only $14 \%(18 / 129)$ were published in indexed journals, the mean time for publication of an article was of 2,7 years. Out of the presented research projects, $68(45 \%)$ were through a competitive fund, $60(40 \%)$ were institutional, $14(9 \%)$ coming from regional (provincial) health directions and $8(5 \%)$ collaborative. The executed budget was of $\$ 5,032,906.62$. The mean amount assigned to each research project was $\$ 33,552.71$ and the cost of each publication was $\$ 279,605.92$; the distribution of the budget according to each study subject was $61 \%$ for communicable diseases, $12 \%$ for non-communicable diseases and $27 \%$ for technological development. The research promotion, development and financing in the Instituto Nacional de Salud during this period have had a decreasing trend, influenced by institutional policy. In order to overcome this situation, not only at an institutional but also at a national level, it is necessary that the State defines its national research policy, respecting the national and regional priorities in health research.
\end{abstract}

Key words: Support of research; Financing, government; Biomedical research; Health science, techonology and innovation management; Peru (source: MeSH NLM).

\section{INTRODUCCIÓN}

La investigación en salud se puede definir como todo proceso dirigido a generar conocimiento sistemático y contrastar hipótesis dentro de las ciencias médicas, aunque no limitado a ellas, por cuanto se extiende además a las ciencias naturales y sociales ${ }^{(1)}$, pero además cumple un rol fundamental en los procesos de formulación y evaluación de políticas, programas e intervenciones en salud. Con esta perspectiva la investigación en salud se considera también parte central de la gobernanza ${ }^{(2)}$.

La investigación de gran calidad es esencial para la equidad, la salud y el desarrollo socioeconómico de los países y para alcanzar los Objetivos de Desarrollo del Milenio relacionados con la salud ${ }^{(3)}$.

\footnotetext{
Oficina General de Investigación y Transferencia Tecnológica; Instituto Nacional de Salud. Lima, Perú.
}

a Enfermera epidemióloga; ${ }^{\text {a }}$ Médico salubrista; ${ }^{c}$ Médico epidemiólogo. 
En el último decenio, la investigación en salud ha suscitado un mayor interés tanto a nivel mundial, como regional; además de haberse producido un notable aumento del financiamiento para la salud (4) y viene experimentando una institucionalización gradual como factor de orientación y cambio en este sector de los países de ingresos medios y bajos ${ }^{(5)}$; debido a que pone a disposición evidencias que permiten mejorar el estado de salud de la población, fundamentar las prioridades y avanzar en la búsqueda de respuestas a los problemas sanitarios, que evolucionan en la medida que cambian las necesidades de la población ${ }^{(6)}$

Si bien es cierto, los hechos demuestran que la investigación en salud es sumamente importante para el desarrollo de un país, porque permite generar evidencias que sustentan las políticas públicas, en el Perú, esta aún no es prioridad para el Estado, pues prevalecen temas de investigación de interés individual que no responden a políticas encaminadas a contribuir o dar solución a los problemas prioritarios de salud nacionales, además, aún no se dispone de una masa crítica suficiente de investigadores con alto nivel de formación, situación que va ligada a la falta de incentivos a los investigadores y a la actividad científica.

El Instituto Nacional de Salud (INS), tiene como uno de sus objetivos funcionales Desarrollar $y$ difundir la investigación científica y tecnológica en salud en los ámbitos nacional y regional; actividad que ha desarrollado desde sus inicios y que a partir del año 2000 , se extendió hacia las regiones, promoviendo y financiando la investigación individual en temas prioritarios de salud, enfocados a enfermedades transmisibles, no transmisibles y de desarrollo tecnológico. El financiamiento para la ejecución de estas investigaciones proviene de fondos del Estado y de recursos directamente recaudados incluidos en el presupuesto del INS. Las investigaciones financiadas podían provenir de necesidades institucionales de investigación, fondos concursables, convenios con diferentes instituciones, apoyo para el desarrollo de tesis y últimamente el desarrollo de programas de investigación.

Este trabajo tiene como objetivo realizar el análisis de la gestión y financiamiento de las investigaciones financiadas por el INS durante el periodo 2004 - 2008, con la finalidad de rescatar las lecciones aprendidas y buscar nuevas estrategias que permitan promover la investigación en salud en el país, así como la generación de nuevos conocimientos y tecnologías para elevar los niveles de salud del individuo, de la familia y de la comunidad.

\section{EL ESTUDIO}

Se realizó un estudio descriptivo, retrospectivo, se usó la base de datos del registro y seguimiento de investigaciones del INS durante el periodo 2004-2008.

Se incluyó aquellas investigaciones que han sido registradas y aprobadas en el INS a partir de enero de 2004 y diciembre de 2008 y que han recibido financiamiento total o parcial del INS, en sus diferentes modalidades (institucional, colaborativa, fondos concursables), se excluyó las tesis de pregrado y posgrado.

Con fines operativos se definió el estado de las investigaciones en la condición en que se encontraban al momento del estudio, definiéndolas como: aprobadas (aquellas que contaban con Resolución Jefatural), ejecutadas (que contando con Resolución Jefatural, iniciaron su ejecución), finalizadas (concluidas con informe final) y publicadas (con artículo científico publicado en revistas biomédicas).

Respecto a la asignación del financiamiento de las investigaciones se clasificó en: institucionales (provenientes de los Centros Nacionales [CN] o Unidades Orgánicas [UO] pertenecientes al INS), colaborativas (desarrolladas conjuntamente con otra/s institución/es a través de un convenio vigente), del fondo concursable (aquellas seleccionadas mediante concurso público [se incluye a los financiados por el Proyecto VIGIA/USAID/MINSA]) y las provenientes de las direcciones regionales de salud (DIRESA/DISA) a solicitud de ellas ${ }^{(7)}$.

Según el tema u objeto de estudio se dividieron las investigaciones en enfermedades transmisibles, no transmisibles y de desarrollo tecnológico.

Los datos referentes al presupuesto fueron proporcionados por la Oficina de Planificación y Presupuesto del INS, en este trabajo solo se hizo referencia al presupuesto ejecutado, no se consideró los costos indirectos que asume la institución como son la infraestructura, el equipamiento, el pago de personal, etc. Por ello estos montos son referenciales, situación que no permite realizar un análisis más fino.

Con relación al financiamiento, para evitar distorsiones debidas a las fluctuaciones cambiarias posibles, todos los montos están expresados en dólares, tomando como referencia el tipo de cambio promedio a diciembre de los años de estudio, se toma como fuente los datos proveídos por el Ministerio de Economía y Finanzas (http://www.mef.gob.pe/INDECO/ tipo_cambio.php). 
Antes del análisis de la base de datos, se procedió a revisar, ordenar, depurar, estandarizar y completar la información de las variables consignadas. Para evaluar la publicación de las investigaciones financiadas, se realizó una búsqueda (Scholar Google, SciELO y MEDLINE) de los investigadores principales y de la temática del estudio en julio de 2010, cuando se ubicó los artículos similares, se comparó con los informes finales para verificar si correspondían al mismo proyecto financiado.

Se realizó el análisis descriptivo en forma tabular y gráfica usando el programa Microsoft Excel $®$

\section{HALLAZGOS}

El Instituto Nacional de Salud entre enero de 2004 y diciembre de 2008, registró en la base de datos 327 investigaciones, aplicando los criterios de inclusión la población de estudio fue de 182 investigaciones, siendo su distribución por años de la siguiente manera: 63 en el 2004; 63 en el 2005; 28 en el 2006; 21 en el 2007 y 7 en el 2008.

En este quinquenio se aprobó 182 investigaciones y se ejecutó 150 (82\%); de los cuales 68 (45\%) fueron del fondo concursable, 60 (40\%) institucionales, 14 (9\%) de las DIRESA o DISA y 8 (5\%) colaborativas (Figura 1).

En este periodo se finalizó el 86\% (129/150) de las investigaciones financiadas; con un comportamiento por año como sigue: $94 \%(51 / 54)$ en el 2004; $94 \%(48 / 51)$ en el 2005; 86\% (18/21) en el 2006, 58\% (11/19) en el 2007 y solo $20 \%$ (1/5) en el 2008. De acuerdo con la asignación para el financiamiento se finalizó el 100\% $(14 / 14)$ de las presentadas por las DIRESA/DISA; el $88 \%(60 / 68)$ de fondo concursable; el $88 \%(7 / 8)$ de las colaborativas y el $80 \%(48 / 60)$ de las institucionales.

Durante este quinquenio se desarrolló cuatro fondos concursables entre los años 2004 - 2007 (Tabla 1) que

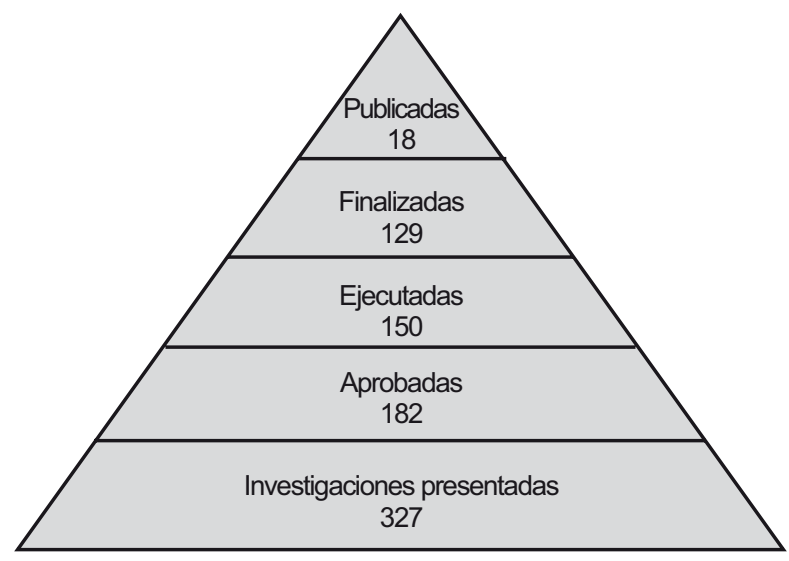

Figura 1. Estado de las investigaciones periodo 2004-2008. Instituto Nacional de Salud
Tabla 1. Investigaciones aprobadas, ejecutadas y finalizadas, según asignación de financiamiento por Instituto Nacional de Salud, 2004-2008.

\begin{tabular}{lcccccc}
\hline Tipo de proyecto & $\mathbf{2 0 0 4}$ & $\mathbf{2 0 0 5}$ & $\mathbf{2 0 0 6}$ & $\mathbf{2 0 0 7}$ & $\mathbf{2 0 0 8}$ & Total \\
\hline $\begin{array}{l}\text { Fondo Concursable } \\
\text { Aprobado }\end{array}$ & 17 & 46 & 6 & 12 & & 81 \\
\hline Ejecutado & 15 & 36 & 6 & 11 & & 68 \\
\hline Finalizado & 15 & 33 & 6 & 6 & & 60 \\
\hline Institucional & & & & & & \\
\hline Aprobado & 25 & 14 & 20 & 6 & 6 & 71 \\
\hline Ejecutado & 23 & 13 & 14 & 5 & 5 & 60 \\
\hline Finalizado & 20 & 13 & 11 & 3 & 1 & 48 \\
\hline DISA /DIRESA & & & & & & \\
\hline Aprobado & 17 & & & & & 17 \\
\hline Ejecutado & 14 & & & & & 14 \\
\hline Finalizado & 14 & & & & & 14 \\
\hline Colaborativo & & & & & & \\
\hline Aprobado & 4 & 3 & 2 & 3 & 1 & 13 \\
\hline Ejecutado & 2 & 2 & 1 & 3 & & 8 \\
\hline Finalizado & 2 & 2 & 1 & 2 & & 7 \\
\hline
\end{tabular}

contribuyeron con el $45 \%$ de las investigaciones aprobadas y $40 \%$ del total de investigaciones finalizadas.

En el periodo 2004-2008, se encontró que nueve regiones concentraron el $80 \%(9 / 24)$ de las investigaciones aprobadas, de las cuales finalizaron en Huánuco el $79 \%$ (15/19), en La Libertad el 80\% (12/15), en Lima el 47\% (7/15) y el $100 \%$ en Tacna (10/10).

En cuanto a las investigaciones institucionales (Tabla 2), el Centro Nacional de Salud Pública (CNSP) ocupa el primer lugar con el 68\% (41/60) de las investigaciones aprobadas, seguido por el Centro Nacional de Salud

Tabla 2. Distribución de las investigaciones aprobadas y finalizadas según Centros Nacionales del INS. 2004-2008.

\begin{tabular}{lcc}
\hline Centro Nacional/ Oficina & Aprobado & Finalizado \\
\hline CNSP & 41 & 34 \\
CENSOPAS & 7 & 4 \\
CENAN & 6 & 6 \\
CENSI & 2 & 2 \\
CNPB & 2 & 1 \\
OGAT/OGITT & 1 & 1 \\
OGIS & 1 & 0 \\
Total & 60 & 48 \\
\hline
\end{tabular}

CNSP: Centro Nacional de Salud Pública; CENSOPAS: Centro Nacional de Salud Ocupacional y Protección del Ambiente para la Salud; CENAN: Centro Nacional de Alimentación y Nutrición; CENSI: Centro Nacional de Salud Intercultural; CNPB: Centro Nacional de Productos Biológicos; OGAT: Oficina General de Asesoría Técnica; OGITT: Oficina General de Investigación y Transferencia Tecnológica; OGIS: Oficina General de Información y Sistemas. 
Ocupacional y Protección del Medio Ambiente para la Salud (CENSOPAS) con $12 \%$ (7/60) y el Centro Nacional de Alimentación y Nutrición (CENAN) con 10\% (6/60).

Las investigaciones en enfermedades transmisibles ocupan el primer lugar con $59 \%$ (las enfermedades más investigadas fueron dengue, TBC y $\mathrm{TBC} / \mathrm{VIH}, \mathrm{VIH}$, malaria y leptospirosis), le sigue las relacionadas con enfermedades no transmisibles con 33\% (problemas nutricionales, cardiovasculares, mortalidad) y las de desarrollo tecnológico con $9 \%$ (referido a técnicas de diagnóstico de laboratorio, sueros, antivenenos y vacunas).

El 14\% (18/129) de las investigaciones terminaron en la publicación de artículos científicos. El promedio de tiempo que demoró publicar un artículo fue de 2,7 años y el rango oscila de 1 a 4 años; el 83\% (15/18) de las publicaciones fueron en revistas nacionales y el $17 \%$ (3/18) en revistas internacionales. Dentro de las publicaciones en revistas nacionales tenemos que el $80 \%$ $(12 / 15)$ fue en la Revista Peruana de Medicina Experimental de Salud Pública (RPMESP) del INS y el resto en la revista Anales de la Facultad de Medicina y la Revista de la Sociedad Peruana de Medicina Interna. Dentro de las internacionales tenemos al American Journal of Tropical Medicine y Archives of Environmental Contamination and Toxicology.

El presupuesto ejecutado porel INS para el financiamiento de las investigaciones durante el periodo 2004-2008 fue de $\$ 5032$ 906,62. En el 2004 se desarrollaron 63 investigaciones, con un presupuesto ejecutado de \$1 308503,59 , mientras que en el 2005, para el mismo número, se ejecutó $\$ 970$ 243,90; en el 2006 para 28 investigaciones se ejecutó $\$ 726775,15$; en el 2007 para 21 investigaciones se ejecutó \$1348 650,28 y en el 2008 para siete investigaciones se asignó un monto de $\$ 678733,70$ (Figura 2).

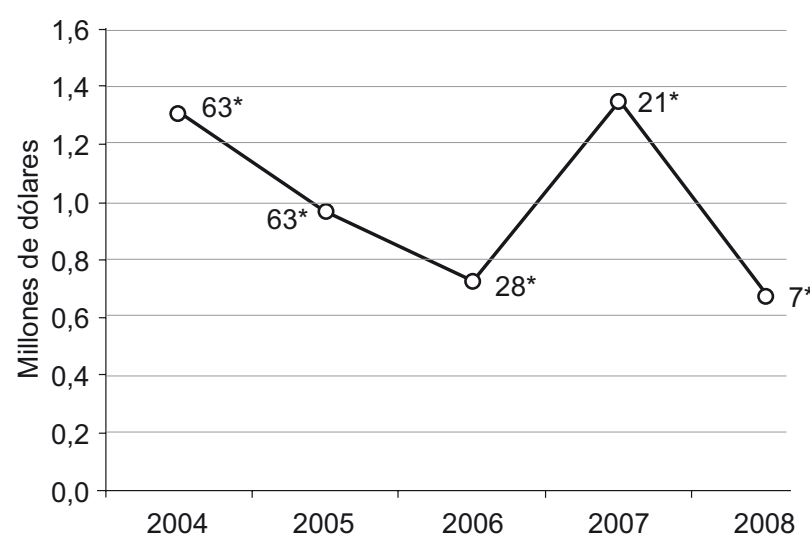

Figura 2. Presupuesto ejecutado por investigaciones en el Instituto Nacional de Salud, Perú 2004-2008.

* Número de investigaciones ejecutadas.

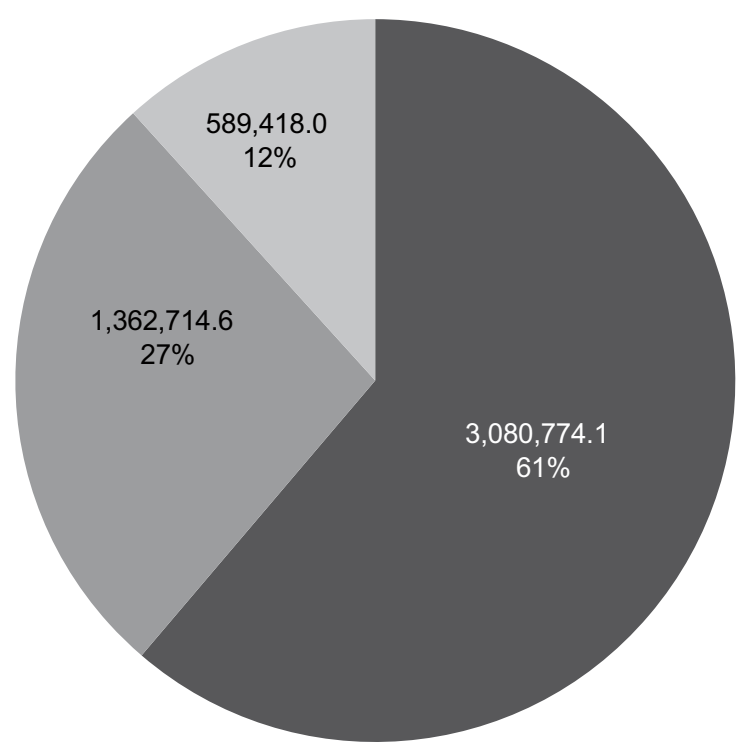

Monto total : $\$ 5032906,7$

- Trasmisibles Desarrollo tecnológico No trasmisibles

Figura 3. Distribución del financiamiento de investigaciones según tema u objeto de estudio. 2004-2008. INS

El financiamiento de las investigaciones fue dividido, según tema u objeto de estudio, en enfermedades transmisibles, no transmisibles y de desarrollo tecnológico, con un presupuesto de $\$ 3080774,1$; $\$ 589417,9$ y $\$ 1362714,6$ respectivamente (Figura 3). El costo unitario promedio asignado por investigación según tema fue de $\$ 35$ 008,8 para enfermedades transmisibles; $\$ 12$ 028,9 para no transmisibles y $\$ 104$ 824,2 para las de desarrollo tecnológico.

Si tenemos en cuenta que se aprobó y financió 150 investigaciones, y se asignó \$5 032 906,6, podríamos decir que cada una costó, en promedio, $\$ 33552,7$. Como finalizaron 129, el costo por investigación culminada sería de $\$ 39014,8$. La publicación, al ser únicamente 18, ascendería a \$ 279 605,9.

\section{DISCUSIÓN}

Durante el quinquenio 2004-2008, se aprobó 182 investigaciones, de ellas se ejecutó 150 (82\%). Las razones por las cuales no se llevó a cabo la totalidad de investigaciones, se debió a que algunas tuvieron observaciones metodológicas y de viabilidad para la ejecución, luego de la aprobación. Se encontró, además, que 21 investigaciones no finalizaron debido a problemas administrativos, tales como demora en la firma de cartas convenio, tardanza en la entrega de cuentas por encargo, problemas en la compra de 
insumos, contratación de servicios, escaso compromiso de las DISA o DIRESA para la ejecución presupuestal, monitoreo y supervisión; así como causas relacionadas con el investigador.

La no culminación de los proyectos aprobados es una situación que se observa incluso en países desarrollados, por ejemplo, cuando se evaluó a 649 proyectos aprobados en 25 Comités de Ética de Francia, $581(90 \%)$ se iniciaron y $80 / 581(14 \%)$ no culminaron (8). Trabajar sobre las causas que llevan a que una investigación aprobada y financiada no inicie o no se culmine, es indispensable para evitar la pérdida de recursos, no solo económicos sino también de tiempos de los investigadores y revisores.

La investigación en las regiones y a nivel nacional es poco promocionada y menos aun financiada, está prácticamente circunscrita a las universidades e institutos de investigación ${ }^{(9)}$. Frente a esta situación, el INS promovió como estrategia de gestión de la investigación, la realización de fondos concursables, modalidad que ha sido desarrollada en países como Argentina y Chile por sus Ministerios de Salud, con presupuesto propio ${ }^{(3,10)}$. Los fondos concursables convocados por el INS, impulsaron el desarrollo de las investigaciones en 24 regiones del país, contribuyendo con el $44,5 \%$ de las investigaciones aprobadas. Los temas que abordaron están en relación con las líneas prioritarias de investigación previamente definidas. El impacto de esta estrategia se vio reflejado en los años 2004 y 2005 pues se registró mayor número de investigaciones, que contribuyeron con el $40 \%$ de investigaciones finalizadas.

Por otro lado, el INS cuenta con seis centros nacionales que realizan investigaciones en mayor o menor medida. El Centro Nacional de Salud Publica desarrolló el $49,4 \%$ de las investigaciones aprobadas, debido a que cuenta con recursos humanos capacitados y una red de laboratorios altamente especializado, encargados de investigar, realizar la vigilancia de enfermedades transmisibles y no transmisibles.

Según el tema u objeto de estudio, predominó la investigación en enfermedades transmibles (59\%) donde el dengue TBC y TBC/VIH, VIH, malaria y leptospirosis fueron los temas más investigados; esto se debe a que nuestro país mantiene un perfil epidemiológico prevalente en enfermedades transmisibles con gran impacto social; mientras que las enfermedades no transmisibles, recién están siendo estudiadas en los últimos años.

En este periodo se ejecutó 13 investigaciones en desarrollo tecnológico, cuyo costo unitario promedio fue tres veces más que las de enfermedades transmisibles y nueve veces más que las de no transmisibles, esto debido a la complejidad en la elaboración y ejecución de este tipo de investigaciones.

Cabezas ${ }^{(11)}$, refiere que los programas de investigación deben vincular la investigación de las áreas biomédica básica, clínica, epidemiológica, salud pública y desarrollo biotecnológico. Esta estrategia de gestión de la investigación fue impulsada a partir del 2007, convocada mediante concurso público en el 2008 y financiada en el 2009. Los temas de los programas de investigación comprendieron accidentes de tránsito e hidatidosis.

La proporción de publicación de los proyectos financiados por el INS es muy baja (14\%; 18/129), incluso comparable con las publicaciones de estudiantes de medicina de un curso de epidemiología de una universidad peruana $(12 \% ; 13 / 110)$ en el periodo $2003-$ 2009 , las cuales son autofinanciadas ${ }^{(12)}$. En contraste, cuando el NIH (National Institutes of Health) de los Estados Unidos evaluó la publicación de los proyectos (grants) que ha financiado, encontró que cada grant en promedio generaba 7,6 artículos publicados en revistas MEDLINE ${ }^{(13)}$.

Estos hallazgos corroboran lo mencionado por diferentes autores, que mencionan que la publicación de investigaciones en nuestro país es precaria ${ }^{(9)}$, que no existe una cultura de publicación ${ }^{(14)} \mathrm{y}$, como bien menciona Pamo ${ }^{(15)}$, se elaboran muchos proyectos, se empiezan algunos, se terminan pocos y se publican menos, lo que se denomina "pirámide de la producción científica nacional", ello se puede apreciar con claridad en la Figura 1.

Al analizar los datos encontramos que los autores de los artículos publicados, por lo general, habían tenido una publicación previa; esto nos indica que debe promoverse capacitaciones en redacción científica, con la finalidad de disminuir esta brecha ${ }^{(16)}$. Este hecho se podría explicar a que la sistematización de la información en un artículo científico, para quien no tiene experiencia, es muy difícil, aunado al escaso tiempo para redactar, escaso interés para publicar, dificultades en el dominio del inglés para publicar en revistas extranjeras, limitación en el análisis de datos, entre otros ${ }^{(14,16)}$.

Cabe señalar que el presupuesto programado para investigación en el sector salud para el periodo de estudio en el Perú, fue de $\$ 18407$ 790, de los cuales $\$ 5032$ 906,62 se ejecutó en el INS, lo cual correspondió al 50\% (\$10 066 190) de lo ejecutado a nivel nacional (disponible en http://ofi.mef.gob.pe/transparencia/ mensual). 
Maceira (1) encontró que en el 2006, en un estudio sobre el financiamiento de investigación en salud con fondos públicos para cinco países de América Latina (Argentina, Bolivia, Chile, Paraguay y Uruguay). Argentina destinó $15,7$ (\$55 424300$)$ y Chile 7,4 (\$26 269 000) veces mayor presupuesto que el Perú (\$3 534 392,51).

El número de investigaciones aprobadas y el presupuesto asignado en el INS durante el 2004 2008, mantuvo una tendencia descendente (Figura 2) la cual estuvo influenciada por la política institucional promovida por cada una de las autoridades que la dirigieron. Si queremos revertir esta situación no solo a nivel institucional sino nacional, es necesario que el Estado defina su Política Nacional de Investigación y respete el financiamiento de las prioridades nacionales y regionales de investigación en salud.

Finalmente, debemos mencionar, que es escasa la bibliografía sobre el tema de financiamiento de las investigaciones a nivel internacional y más aun a nivel nacional, situación que no nos ha permitido comparar nuestros resultados con otras realidades.

\section{AGRADECIMIENTOS}

A la Oficina General de Asesoría Técnica, en especial al Sr. economista Miguel Felices Mendoza.

\section{Conflictos de Interés}

Los autores son parte de la Oficina de Investigación y Transferencia Tecnológica del Instituto Nacional de Salud que es responsable de los procesos descritos en este artículo.

\section{REFERENCIAS BIBLIOGRÁFICAS}

1. Maceira D, Paraje G, Aramayo F, Duarte Masi S, Sánchez D. Financiamiento público de la investigación en salud en cinco países de América Latina. Rev Panam Salud Publica. 2010;27(6):442-51.

2. De los Ríos R. Gobernanza de la investigación función esencial de la salud pública. Elementos para la discusión. Washington DC: OPS; 2005.

3. World Health Organization. Report of the Task Force on Health Systems Research. The Millennium Development Goals will not be attained without new research addressing health system constraints to delivering effective interventions. Geneva: WHO; 2005.
4. Organización Panamericana de la Salud/Organización Mundial de la Salud. $49 .^{\circ}$ Consejo Directivo: Política de investigación para la salud. CD49/10. Washington DC: OPS/OMS; 2009.

5. González-Block MA, Mercado FJ, Ochoa H, Rivera H, Idrovo AJ. Utilización de la investigación por gestores de salud: desarrollo y validación de una herramienta de autodiagnóstico. Salud Publica Mex. 2008;50(6):490-97.

6. Alger J, Becerra-Posada F, Kennedy A, Martinelli E, Cuervo LG, Grupo Colaborativo de la Primera Conferencia Latinoamericana de investigación e Innovación para la Salud. Sistemas nacionales de investigación para la salud en América Latina: una revisión de 14 países. Rev Panam Salud Publica. 2009; 26(5): 447-57.

7. Instituto Nacional de Salud. Directiva para la presentación, aprobación, ejecución, seguimiento y finalización de proyectos de investigación observacionales. Lima: INS; 2010.

8. Decullier E, Lheritier V, Chapuis F. Fate of biomedical research protocols and publication bias in France: retrospective cohort study. BMJ. 2005;331(7507):19.

9. Cabezas C. Rol del Estado en la investigación científica en salud y transparencia en la información. Rev Peru Med Exp Salud Publica. 2006;23(4):275-83.

10. Maceira D, Peralta M. El financiamiento público de la investigación en salud en Argentina. Desarrollo Econ. 2008;48(189): 61-84.

11. Cabezas C. Programas de investigación: una alternativa integral e incluyente para enfrentar los problemas de salud pública. Rev Peru Med Exp Salud Publica. 2006;23(3):147-48.

12. Alarcón-Villaverde J, Romaní f, Gutiérrez C. Publicaciones científicas estudiantiles producidas en el curso de epidemiología de la Facultad de Medicina de la Universidad Nacional Mayor de San Marcos durante el periodo 20032009. An Fac Med (Lima). 2010;71(2):111-16.

13. Druss BG, Marcus SC. Tracking publication outcomes of National Institutes of Health grants. Am J Med. 2005;118(6):658-63.

14. Gutierrez C, Mayta P. Publicación desde el pregrado en Latinoamérica: importancia, limitaciones y alternativas de solución. CIMEL. 2003;8(1):54-60.

15. Pamo O. Estado actual de las publicaciones periódicas científicas médicas del Perú. Rev Med Hered. 2005;16(1):65-73.

16. Molina-Ordoñez J, Huamaní C, Mayta-Tristán P. Apreciación estudiantil sobre la capacitación universitaria en investigación: estudio preliminar. Rev Peru Med Exp Salud Publica. 2008;25(3):325-29.

\author{
Correspondencia: Gladys Garro \\ Dirección: Cápac Yupanqui 1400, Lima11, Perú. \\ Teléfono: (511) 617-6200 \\ Correo electrónico: ggarro@ins.gob.pe
}

